\title{
Socio-economic status and outcomes for patients with age-related macular degeneration
}

\author{
Pradnya More $\mathbb{B}^{1} \cdot$ Hussein Almuhtaseb ${ }^{2} \cdot$ Dianna Smith $^{3} \cdot$ Simon Fraser $\mathbb{D}^{4} \cdot$ Andrew J. Lotery $\mathbb{D}^{1}$
}

Received: 18 June 2018 / Revised: 15 January 2019 / Accepted: 7 February 2019 / Published online: 11 March 2019

(c) The Royal College of Ophthalmologists 2019

\begin{abstract}
Objectives To explore disparities in severity of baseline disease, treatment completion, and treatment outcomes among patients with wet age-related macular degeneration (AMD) receiving anti-vascular endothelial growth factor therapy by socio-economic status (SES) and distance from home to hospital.

Study design Retrospective cohort study.

Methods Data from clinic records of 756 wet AMD patients receiving treatment for wet AMD with aflibercept between May 2013 and Jan 2017 were obtained. Area SES (using Index of Multiple Deprivation (IMD) 2015) and distance from hospital (dichotomized $>=10$ vs. $<10 \mathrm{~km}$ ) were derived from anonymized postcodes. Univariate and multivariable logistic regression models were used to identify associations of area deprivation and distance from hospital at baseline-with visual acuity (VA) at baseline - treatment completion, and treatment outcome.

Results Living in the most deprived compared with less deprived areas was associated with a significantly higher risk of presenting with severe reduction in VA $(\mathrm{OR}=3.59 ; 95 \% \mathrm{CI}=1.39-9.27 ; P=.01)$. This association was maintained after adjustment for age, gender, and distance from hospital. On univariate analysis, delayed treatment completion was more likely in those living in most deprived areas $(\mathrm{OR}=2.80 ; 95 \% \mathrm{CI}=1.21-6.47 ; P=.04)$, though this association was attenuated after adjustment for age, gender, and distance from hospital. No association was observed between SES and treatment outcomes or between distance from hospital and baseline VA, treatment completion or treatment outcome.

Conclusion This study found poorer baseline VA among people with wet AMD from more deprived areas. This work suggests a need for earlier identification of AMD among more deprived populations.
\end{abstract}

Supplementary information The online version of this article (https:// doi.org/10.1038/s41433-019-0393-3) contains supplementary material, which is available to authorized users.

Andrew J. Lotery

A.J.LOTERY@SOTON.AC.UK

1 Faculty of Medicine, University of Southampton, Southampton, UK

2 University Hospital Southampton NHS Foundation Trust, Southampton, UK

3 Department of Geography, University of Southampton, Southampton, UK

4 Academic Unit of Primary Care and Population Sciences, Faculty of Medicine, Southampton General Hospital, University of Southampton, Southampton, UK

\section{Introduction}

In UK, there are approximately 1.8 million people living with substantial sight loss, of which $16.7 \%$ are due to agerelated macular degeneration (AMD) [1]. There are around 70,000 newly diagnosed cases of late (dry and wet) AMD every year in UK [2]. Older age is the most important determinant of AMD [3]. In addition, there are other modifiable risk factors, particularly smoking [3]. Due to these determinant factors, both incidence and prevalence are likely to rise globally $[4,5]$. AMD is the biggest single cause of sight loss in UK [1]. Along with sight loss, AMD impact includes depression, social isolation, falls, increased cost due to assistive needs and home support [6-9]. The disease adversely impacts quality of life and creates a cost burden [10]. In neovascular AMD, rapid treatment with intravitreal injection of anti-VEGF monoclonal antibodies such as aflibercept, ranibizumab, or bevacizumab can prevent further vision loss and in some cases reverse vision 
loss [11]. However, delays in treatment can lead to permanent visual loss. Early identification of risk factors of AMD combined with advances in treatment has meant that AMD is included in UK as a key public health indicator [12]. Smoking, a risk factor for late AMD, is also associated with lower socioeconomic status (SES) [13].

Little is known about the association of SES and AMD or the effect of distance from hospital as a proxy for access to treatment. One study conducted in Birmingham, UK suggested that people living in most deprived areas had more severe visual outcomes in AMD, whereas another study in Norfolk demonstrated the association of AMD with people living in affluent areas [14, 15]. However other studies in Australia and UK have shown no association between SES and AMD [16, 17]. Studies conducted in France and Turkey showed a positive association of greater distance with increased number of loss of treatment followups of AMD, whereas in a Polish study place of residence had no effect on treatment pathway of AMD [18-20]. Although a systematic review by Charlotte Kelly states that a relationship between travel time/distance and worse health outcome cannot be ruled out; as travel time is not available in our study, we have only considered travel distance [21].

In view of these conflicting prior studies, this study explored if there was an association between SES and severity of AMD at presentation to hospital and whether SES and distance from hospital were associated with treatment completion and treatment outcome.

\section{Methodology}

Patients included in this retrospective cohort study were treated according to National Institute for Health and Care Excellence (NICE) guidance [22]. Neovascular AMD was diagnosed by ophthalmologists based on clinical assessment, fluorescein angiography and optical coherence tomography [23]. Data from 756 1st-treated eyes of 756 patients were included. Only data from the 1st-treated eyes with neovascular AMD with aflibercept were analyzed in our retrospective cohort study.

The target population for this study was wet AMD patients aged over 50 years who received treatment by injection of aflibercept using a locally developed care pathway [24]. NICE guidelines were followed where patients with best corrected visual acuity (VA) between 0.3 (70 ETDRS letters; mild visual impairment) and 1.2 (25 ETDRS letters; blindness) on a Logmar Vision Chart were treated [23, 25]. However some patients were treated with vision outside these criteria for a variety of reasons including it being felt that there were other reasons other than their AMD causing reduced vision such as cataract or because they met NICE criteria at start of treatment and treatment was continued even if vision had deteriorated during treatment.

Secondary data analysis was conducted by using AMD patients identified from existing hospital patient records and postcode containing data was anonymized prior to analysis. The study population was purposively sampled as all wet AMD patients who received their first injection of aflibercept between May 2013 and Jan 2017.

\section{Demographic information}

Age, sex, ethnicity, current diabetic status, and self-reported smoking was identified from patient records. Age was categorized into two groups: Older people (50 to 85 years) and oldest old ( $>85$ years). Ethnicity was categorized as British, any other white, Mixed ethnicity and patients who have not stated their ethnicity. Diabetes included patients with no diabetes, Type 1 diabetes, Type 2 diabetes, diabetes not known, and smoking includes ex-smoker, smoker, and never smoked.

SES was defined by area deprivation using the 2015 Index of Multiple Deprivation (IMD). IMD provides a relative measure of material and social deprivation for small areas in England. Lower Super Output Areas (LSOA) have an average population of 1500 and are ranked on seven domains: income, employment, health deprivation and disability, education skills and training, barriers to housing and services, crime and living environment [26]. Anonymized postcodes were assigned the correct LSOA and corresponding IMD Decile using the online GeoConvert Tool provided by the official website of UK Data Services [27]. IMD was categorized into quintiles where the 1st quintile is most deprived and the 5th quintile least deprived.

The median distance (Euclidean, as the crow flies) between home and SEU was calculated for each postcode using ArcGIS 10.5. Median distance was dichotomized as 0-10 km (Kilometers) (near distance), >10 km (greater distance) [28].

\section{Outcome variables}

- Severity of wet AMD was defined by vision impairment at baseline, i.e., date of first presentation at the eye unit.

This was further dichotomized using standard guidelines Mild-Moderate ability to see equal or more than 35 letters on a standard ETDRS chart [25, 29].

Severe ability to see less than 35 letters on a standard ETDRS chart.

- Treatment process was assessed as delay in completion of treatment if patients failed to complete the treatment course in the recommended period of 11 months in the first year. 
- Treatment outcome after aflibercept therapy was assessed by change in VA from baseline to latest follow-up after treatment completion, dichotomized as:

Deterioration of vision: $>=$ loss of 15 letters on ETDRS chart.

Maintain or improvement of vision: <loss of 15 letters on ETDRS chart.

\section{Analysis strategy for the study}

All analyses were conducted using IBM SPSS Statistics for Windows, Version 22.0. The pre-specified level of significance chosen for the study was 0.05. All statistical analysis in SPSS is by default 2-tailed. The Shapiro-Wilk test of normality was run to investigate the distribution of data which was found to be skewed.

Descriptive statistics were used to identify sociodemographic and clinical characteristics of whole study $(n=756)$ and subset population $(n=524)$.

As the data was skewed, the variables with continuous data were explored by using median (measure of central tendency) and range (estimation of variance). To compare the medians across the quintiles of deprivation the nonparametric Kruskal-Wallis test was conducted whereas to compare the groups based on distance a non-parametric Mann-Whitney $U$ test was conducted. In addition, frequency (percentage), cross tabulation, and chi-square test along with Fishers' Exact test were used for categorical variables to compare different groups.

In addition, associations between independent variables, viz., SES, distance from home to eye unit, age, gender, and dependent variable, viz., severity of BVA, treatment process and treatment outcomes (change in vision from baseline to latest follow-up), were further investigated using univariate and multivariate logistic regression models; where the results were interpreted as odds ratio (relative measure of effect to compare study groups) and confidence interval (an interval estimate combined with probability statement).

\section{Results}

Seven hundred and fifty six patients were identified from the hospital records where median age was 85 years (range 54 $100)$; and 499 patients $(66 \%)$ were female. The age of patients was well-matched across the quintiles. There were a high proportion of white British patients $(93.1 \%)$. Diabetes status was missing from $67.9 \%$ patients. The rest of the study population were mainly non-diabetic $157(20.8 \%)$ with some evidence of type $2(10.7 \%)$ and type 1 diabetes $(0.3 \%)$. Smoking status was un-reported in $638(84.4 \%)$ patients in
Table 1 Socio-demographic and clinical characteristics (whole study and subset population)

\begin{tabular}{|c|c|c|}
\hline & Full & Subset \\
\hline Total number of patients $N(\%)$ & 756 & 524 \\
\hline Age in years-median (Range) & $85(54-100)$ & 85 (54-99) \\
\hline \multicolumn{3}{|l|}{ Sex } \\
\hline Female $N(\%)$ & $499(66)$ & $355(67.7)$ \\
\hline Male $N(\%)$ & $257(34)$ & $169(32.3)$ \\
\hline \multicolumn{3}{|l|}{ Ethnicity $N(\%)$} \\
\hline British & $704(93.1)$ & $493(94.1)$ \\
\hline Any other White & $10(1.3)$ & $4(0.8)$ \\
\hline Mixed ethnicity & $32(1.3)$ & $7(1.3)$ \\
\hline Not stated & 4.2 & $20(3.8)$ \\
\hline \multicolumn{3}{|l|}{ Diabetes $N(\%)$} \\
\hline No diabetes & $157(20.8)$ & $113(21.6)$ \\
\hline Type 1 diabetes & $2(0.3)$ & $1(0.2)$ \\
\hline Type 2 diabetes & $81(10.7)$ & $58(11.1)$ \\
\hline Diabetes but type not known & $3(0.4)$ & $2(0.4)$ \\
\hline Status not known (missing) & 67.9 & 66.8 \\
\hline Smoking $N(\%)$ & $638(84.4)$ & \\
\hline Ex-smoker & $34(4.5)$ & $22(4.2)$ \\
\hline Smoker & $48(6.3)$ & $40(7.6)$ \\
\hline Never smoked & $36(4.8)$ & $23(4.4)$ \\
\hline \multicolumn{3}{|c|}{ Index of multiple deprivation $N(\%)$} \\
\hline Quintile 1 (most deprived) & $34(4.49)$ & $22(4.20)$ \\
\hline Quintile 2 & $86(11.38)$ & $60(11.45)$ \\
\hline Quintile 3 & $134(17.72)$ & $90(17.18)$ \\
\hline Quintile 4 & $186(24.60)$ & $126(24.04)$ \\
\hline Quintile 5 (least deprived) & $316(41.80)$ & $226(43.13)$ \\
\hline \multicolumn{3}{|l|}{ Distance (home to eye unit) $N(\%)$} \\
\hline Near $\left(<10 \mathrm{~km}^{\mathrm{a}}\right)$ & $413(54.62)$ & $288(54.96)$ \\
\hline Far $(>10 \mathrm{~km})$ & $343(45.37)$ & $236(45.03)$ \\
\hline
\end{tabular}

${ }^{\mathrm{a}} \mathrm{km}$ kiolmeters

these routine data. Of the remaining 116 patients, 34 (4.5\%) were ex-smokers, 36 (4.8\%) were non-smokers and 48 (6.3\%) were current smokers. Descriptive statistics showed that a much smaller number of patients receiving aflibercept injections, 34 (4.49\%) were from quintile 1 (the most deprived area) compared with quintile 5 (the least deprived area) where $316(41.8 \%)$ received treatment. Of the 756-total population, $413(54.62 \%)$ patients were living in postcode areas which were closer than $10 \mathrm{~km}$ to the eye unit (Table 1).

Some clinical data was missing from this retrospective cohort. Therefore, a sub-population of 524 patients with complete data including VA outcomes, treatment process, and socio-demographic characteristics were used to explore the associations of treatment outcomes (Fig. 1). While the results of this selected population should be interpreted with caution, both the population set (756 and 


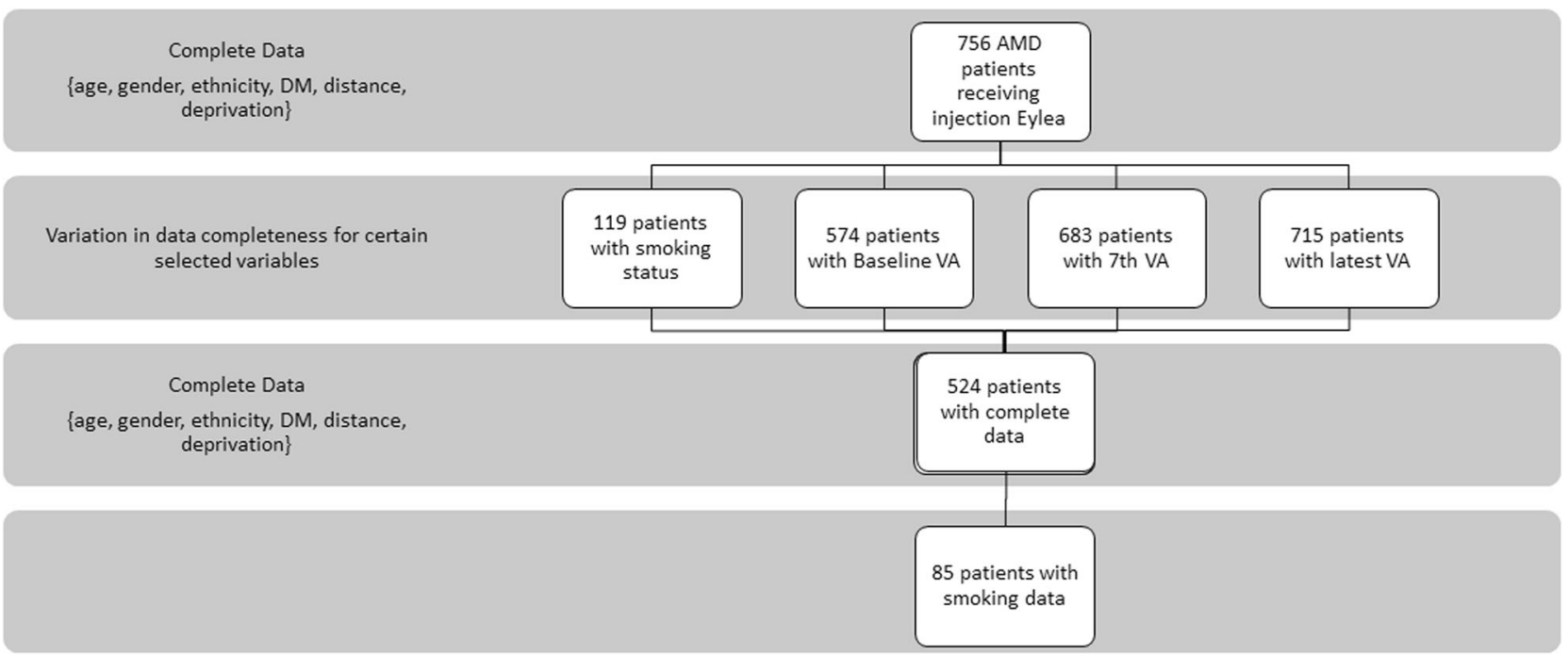

Fig. 1 A flowchart showing cohort entry in the study. AMD age-related macular degeneration, VA visual acuity

Table 2 Associations of AMD severity (severe vs. mild) at baseline

\begin{tabular}{|c|c|c|c|c|c|c|}
\hline & \multicolumn{3}{|l|}{ Univariate } & \multicolumn{3}{|l|}{ Multivariate } \\
\hline & Odds ratio & $(95 \% \mathrm{CI})$ & $P$-value & Odds ratio & $(95 \% \mathrm{CI})$ & $P$-valuc \\
\hline Age (per year increase) & 1.02 & $0.99-1.06$ & 0.23 & 1.03 & $0.99-1.06$ & 0.15 \\
\hline \multicolumn{7}{|l|}{ Gender } \\
\hline Male & Ref. & & & Ref. & & \\
\hline Female & 0.67 & $0.39-1.13$ & 0.13 & 0.63 & $0.37-1.09$ & 0.09 \\
\hline \multicolumn{7}{|l|}{ Deprivation } \\
\hline Quintile 5 (least deprived) & Ref. & & 0.017 & Ref. & & 0.006 \\
\hline Quintile 1 (most deprived) & 3.59 & $1.39-9.27$ & & 4.07 & $1.50-11.0$ & \\
\hline Quintile 2 & 0.45 & $0.15-1.32$ & & 0.47 & $0.15-1.41$ & \\
\hline Quintile 3 & 0.88 & $0.42-1.83$ & & 0.94 & $0.44-1.99$ & \\
\hline Quintile 4 & 0.72 & $0.36-1.44$ & & 0.34 & $0.34-1.36$ & \\
\hline
\end{tabular}

Distance

Near to hospital $\left(<=10 \mathrm{~km}^{\mathrm{a}}\right) \quad$ Ref

Far from hospital $(>10 \mathrm{~km}) \quad 1.13$

$\begin{array}{lll}0.68-1.89 & 0.63 \quad 1.25\end{array}$

$0.72-2.18$

0.43

$O R$ odds ratio, $A M D$ age related macular degeneration

Statistically significant p-values are bold

${ }^{\mathrm{a}} \mathrm{km}$ kilometer

524) showed similar proportions for most characteristics (Table 1).

BVA (median number of letters read on an ETDRS Chart) varied across the quintiles of deprivation with relatively poorer vision $(46.5 ; 24-74)$ among people living in most deprived areas as compared to less deprived areas $(57 ; 3-86)$. However the variation was not statistically significant $(P$ $=.07)$. A statistically significant higher proportion of severe AMD cases occurred in quintile 1 compared to other quintiles; this suggests an association between greater deprivation and severe visual impairment at baseline. No statistically significant difference was seen between BVA and distance from the eye unit $(P=.63)$ (Appendix A).

A positive univariate association was found between greater AMD severity and greater area deprivation at baseline $(\mathrm{OR}=3.59 ; 95 \% \mathrm{Cl}=1.39-9.27 ; P=.017)$. This association was maintained on multivariable analysis adjusting for age, gender, and distance from eye unit $\quad(\mathrm{OR}=4.07 ; \quad 95 \% \quad \mathrm{Cl}=1.50-11.0 ; \quad P=.006)$. Although univariate results were not statistically significant $(\mathrm{OR}=1.13 ; 95 \% \mathrm{CI}=0.68-1.89 ; \quad P=.63)$, greater distance from home was a key exposure of 
Table 3 Delay in completion of treatment

\begin{tabular}{|c|c|c|c|c|c|c|}
\hline & \multicolumn{3}{|l|}{ Univariate } & \multicolumn{3}{|l|}{ Multivariate } \\
\hline & Odds ratio & $(95 \% \mathrm{CI})$ & $* P$-value & Odds ratio & $(95 \% \mathrm{CI})$ & $* P$-value \\
\hline Age (per year increase) & 1.00 & $0.97-1.03$ & 0.99 & 1.00 & $0.97-1.03$ & 0.99 \\
\hline \multicolumn{7}{|l|}{ Gender } \\
\hline Male & Ref. & & & Ref. & & \\
\hline Female & 1.27 & $0.83-1.96$ & 0.28 & 1.32 & $0.86-2.05$ & 0.21 \\
\hline \multicolumn{7}{|l|}{ Deprivation } \\
\hline Quintile 5 (least deprived) & Ref. & & 0.049 & Ref. & & 0.066 \\
\hline Quintile 1 (most deprived) & 2.80 & $1.21-6.47$ & & 2.61 & $1.10-6.19$ & \\
\hline Quintile 2 & 1.64 & $0.85-3.17$ & & 1.58 & $0.81-3.09$ & \\
\hline Quintile 3 & 1.29 & $0.71-2.33$ & & 1.26 & $0.68-2.30$ & \\
\hline Quintile 4 & 1.86 & $1.12-3.09$ & & 1.89 & $1.13-3.13$ & \\
\hline \multicolumn{7}{|l|}{ Distance } \\
\hline Near to hospital $\left(<=10 \mathrm{~km}^{\mathrm{a}}\right)$ & Ref. & & & Ref. & & \\
\hline Far from hospital $(>10 \mathrm{~km})$ & 0.77 & $0.51-1.15$ & 0.21 & 0.85 & $0.56-1.30$ & 0.46 \\
\hline
\end{tabular}

interest in this study and therefore included in the multivariable model. No association was identified between greater distance from home to eye unit and poorer vision at baseline (Table 2).

Across the quintiles of deprivation, the findings were as follows.

\section{Treatment process}

Delayed completion of aflibercept injection course (beyond 11 months). There was a statistically significant difference ( $P$ $=.04$ ) in patients across groups. A higher proportion of patients $(26.5 \%)$ who could not complete treatment in the recommended period (11 months) lived in the most deprived areas (Appendix A). The data further showed no difference in patients living near or far from the eye unit, in terms of delayed completion of aflibercept treatment $(P=.20)$.

In univariate logistic regression, there was an association between greater deprivation and delayed completion in treatment $(\mathrm{OR}=2.80 ; 95 \% \quad \mathrm{Cl}=1.21-6.47 ; \quad P=.04)$. However after adjusting for age, gender, and distance this association was not maintained $(\mathrm{OR}=2.61 ; 95 \% \mathrm{Cl}=$ 1.10-6.19; $P=.06)$. No association was seen between distance and delayed completion of treatment $(\mathrm{OR}=0.77$; $95 \% \mathrm{Cl}=0.51-1.15 ; P=.21$ ) (Table 3$)$.

\section{Treatment outcome}

Although the median gain in the vision after follow-up was high in the most deprived quintile as compared to the least; it was not statistically significant $(P=.88)$ (appendix A).
A higher proportion of patients with poor vision (loss $>=$ 15 letters) after completion of treatment resided in the most deprived areas $(22.7 \%)$ compared to other groups (appendix A). However the difference was not statistically significant $(P=.57)$. In univariate logistic regression, there was no association between greater deprivation and treatment outcomes $(\mathrm{OR}=0.69 ; 95 \% \mathrm{Cl}=0.24-1.98 ; \quad P=.61)$. No association was seen between distance and treatment outcomes $(\mathrm{OR}=0.84 ; 95 \% \mathrm{Cl}=0.52-1.34 ; P=.46)$ (Table 4).

\section{Discussion}

This study investigated the associations between SES and greater distance from home to hospital and severity, treatment processes and outcomes of wet AMD patients, receiving aflibercept injections in SEU between May 2013 and January 2017. The study found that patients living in the most deprived areas had more severe visual impairment when they first visited the eye unit, compared to less deprived areas.

This suggested late presentation of patients after disease progression had already occurred. There was some evidence of greater delay in completion of treatment process among people from most deprived areas, but this association was not maintained after adjustment for other factors. No statistically significant association was found between SES and treatment outcomes. However, a higher proportion of patients in most deprived areas did not complete treatment within the recommended period ( $26.5 \%$ vs. $11.4 \%$ in the least deprived).

While vision improved in the most socially deprived group, it never attained the same level as patients from least 
Table 4 Negative treatment outcome (deteriorated visual acuity after latest follow-up)

\begin{tabular}{|c|c|c|c|c|c|c|}
\hline & \multicolumn{3}{|l|}{ Univariate } & \multicolumn{3}{|l|}{ Multivariate } \\
\hline & Odds ratio & $(95 \% \mathrm{CI})$ & $P$-value & Odds ratio & $(95 \% \mathrm{CI})$ & $P$-value \\
\hline Age (per year increase) & 0.99 & $0.96-1.02$ & 0.59 & 0.99 & $0.96-1.02$ & 0.53 \\
\hline \multicolumn{7}{|l|}{ Gender } \\
\hline Male & Ref. & & & Ref. & & \\
\hline Female & 1.18 & $0.72-1.94$ & 0.51 & 1.22 & $0.74-2.02$ & 0.44 \\
\hline \multicolumn{7}{|l|}{ Deprivation } \\
\hline Quintile 5 (least deprived) & Ref. & & 0.61 & Ref. & & 0.57 \\
\hline Quintile 1 (most deprived) & 0.69 & $0.24-1.98$ & & 0.63 & $0.21-1.8$ & \\
\hline Quintile 2 & 1.82 & $0.73-4.53$ & & 1.73 & $0.68-4.39$ & \\
\hline Quintile 3 & 1.01 & $0.53-1.95$ & & 0.96 & $0.49-1.88$ & \\
\hline Quintile 4 & 1.21 & $0.66-2.23$ & & 1.24 & $0.67-2.29$ & \\
\hline \multicolumn{7}{|l|}{ Distance } \\
\hline Near to hospital $\left(<=10 \mathrm{~km}^{\mathrm{a}}\right)$ & Ref. & & & Ref. & & \\
\hline Far from hospital (>10 km) & 0.84 & $0.52-1.34$ & 0.46 & 0.84 & $0.51-1.38$ & 0.48 \\
\hline
\end{tabular}

deprived areas. Late presentation, seen more commonly in people from more deprived areas, resulted in worse vision at year 1 . No associations were identified between distance from home to eye unit and wet AMD severity, treatment processes, or outcomes. Greater distance did not appear to restrict patients from seeking timely treatment.

Previous studies have explored associations between SES and other eye conditions, including glaucoma and diabetic retinopathy, but few have examined the relationship with AMD [30-32]. Variation in findings may reflect variation in SES measure used and study size. Some, as with our study, used area-based indices that include several weighted domains, such as IMD and Scottish IMD, or estimates of area income [14-16, 33, 34]. Others used individual SES measures such as education, income, and occupation [35-37]. Our results are consistent with many of these studies: showing most deprived patients presented with severe VA at baseline, as compared to less deprived patients. One Scottish study did not demonstrate association between higher area deprivation and poor visual acuity, though numbers were small $(n=240)$ [17]. In our study, median BVA, when measured as the number of letters read on ETDRS chart, did not show a significant difference across the quintiles of deprivation. Insufficient numbers of participants from the most deprived areas may mean that our study was underpowered to detect such a difference.

The positive association between AMD severity and SES may relate to higher prevalence of adverse lifestyle factors in more deprived groups. For example, smoking is known to be more prevalent in lower SES groups and may have mediated the relationship between SES and AMD. Unfortunately we were unable to assess this due to missing smoking data [3]. Poor diet leading to lower levels of serum carotenoids and lutein zeaxanthin (important components of macular pigments, playing a crucial role in visual function) may be another mediator [38, 39]. Greater lack of awareness of AMD, and the fact that its associated sight loss is painless, may contribute to later presentation among more deprived groups [40]. Moreover, poorer access to health services among older people, financial constraints, transport issues, and low health literacy can be barriers to initiate preventive examination [41, 42].

Unlike our UK-based study, greater distance from home to hospital was reported as influencing referral refusals in an Australian study of a new low-vision rehabilitation service [43]. Similarly, a US based study among Medicare beneficiaries, showed reduction in frequency of eye examinations due to greater distance from an ophthalmologist [44].

To the best of our knowledge, no prior studies have examined the relationship between distance from home to hospital and treatment outcomes of aflibercept therapy in people with AMD, although the relationship between ranibizumab injection outcomes and distance has been explored $[18,19]$. Both drugs are anti-VEGF therapies with similar NICE guidelines for administration and duration [23]. Our results differ from the ranibizumab studies [18, 19], where greater distance was associated with poor patient adherence to follow up and treatment. It is consistent with a Polish study where place of residence was not associated with AMD outcomes [20]. One explanation for these differences may be our sampling method. This resulted in exclusion of eligible wet AMD patients who refused aflibercept treatment for unknown reasons. The lack of association between SES or distance and visual outcomes after aflibercept treatment 
suggests an equitable appointment system that is unbiased towards postcode areas. Additionally, data validity and reliability of key variables were maintained using standard protocols and SES and distance were calculated using standard tools (IMD and Geographic Information System (ArcGIS software)). Our study sample size (756) was also relatively high compared to previous wet AMD studies.

This study has strengths and limitations. Strengths included the sample size and the availability of IMD data to allow assessment of SES. Consistency of treatment was also a strength, with all patients diagnosed with wet AMD receiving the same mandated treatment pathway of 7 aflibercept injections in the 11 months post diagnosis. However, the data related exclusively to neovascular AMD patients, a larger set of patients with early AMD, dry AMD and those undergoing different treatment pathways were excluded. Selection bias may also have arisen from the need to restrict the analyses to those with complete data. This restriction also meant we were unable to derive prevalence of neovascular AMD patients as a proportion of all AMD patients. Missing data limited our ability to assess smoking as a confounding or mediating factor.

Early detection and prevention can help reduce illness and overall cost burden, improving the quality of life of older people [10]. We explored SES and distance as barriers to early detection, presentation, and treatment in people with neovascular AMD. Our findings imply that area deprivation is an important risk factor for late presentation of wet AMD (and more important than distance from hospital). This may guide service providers to consider innovative methods to reach people living in such areas and those living further away from eye unit. Such targeted service provision may improve treatment outcomes of this devastating disease.

\section{Summary}

\section{What was known before}

- Limited knowledge of association of socio-economic status and AMD; whatever is known has mixed outcomes.

- Limited knowledge of distance from hospital and AMD.

\section{What this study adds}

- First study which looks for the association of both factors together, socio-economic status and distance from hospital, with severity of AMD and treatment outcomes of injection Aflibercept.

- The study population was relatively larger compared to previous studies.
Acknowledgements We acknowledge Dr. Penny Nestel, Dr. Emma Parsons, and Paula Sands for helpful guidance for this project.

\section{Compliance with ethical standards}

Ethical approval Ethics approval was sought and was granted by Faculty of Medicine for data that is anonymized, as well as postcode data to derive IMD and distance (Ethics and Research Governance Online ID = 29347).

Conflict of interest The authors declare that they have no conflict of interest.

Publisher's note: Springer Nature remains neutral with regard to jurisdictional claims in published maps and institutional affiliations.

\section{References}

1. Access Economics. Future cost of sight loss UK (1): Economic impact of partial sight and blindness in the UK adult population. http://www.rnib.org.uk/knowledge-and-research-hub/researchreports/general-research/future-sight-loss-uk-1. (2009). Accessed 10 Nov 2017.

2. Owen CG, Jarrar Z, Wormald R, Cook DG, Fletcher AE, Rudnicka AR. The estimated prevalence and incidence of late stage age related macular degeneration in the UK. Br J Ophthalmol. 2012;96:752-6.

3. Chakravarthy U, Wong TY, Fletcher A, Piault E, Evans C, Zlateva $\mathrm{G}$, et al. Clinical risk factors for age-related macular degeneration: a systematic review and meta-analysis. BMC Ophthalmol. 2010;10:31.

4. National Population Projections. Office for National Statistics. https://www.ons.gov.uk/peoplepopulationandcommunity/popula tionandmigration/populationprojections/bulletins/nationalpopula tionprojections/2015-10-29. (2017). Accessed 10 Oct 2017.

5. Wong WL, Su X, Li X, Cheung CM, Klein R, Cheng CY, et al. Global prevalence of age-related macular degeneration and disease burden projection for 2020 and 2040: a systematic review and meta-analysis. Lancet Glob Heal. 2014;2:106-16.

6. Macular Society. Age-related macular degeneration: collaborating to find a cure. Collaborating to find a cure Accessible FINAL.pdf. https://www.macularsociety.org/sites/default/files/downloads/ AMD. (2016). Accessed 10 Oct 2017.

7. McManus S, Lord C. Circumstances of people with sight loss. Secondary analysis of Understanding. Society the Life and Opportunities. National Centre for Social Research. http://www. rnib.org.uk/sites/default/files/Circumstances_sight_loss_report. pdf. (2012). Accessed 11 Nov 2017.

8. Owen CG, Fletcher AE, Donoghue M, Rudnicka AR. How big is the burden of visual loss caused by age related macular degeneration in the United Kingdom? Br J Ophthalmol. 2003;87:312-7.

9. Scuffham P, Legood R, Wilson E, Kennedy-Martin T. Incidence and cost of injurious falls associated with visual impairment. Vis Impair Res. 2002;4:1-14.

10. Lotery $\mathrm{A}, \mathrm{Xu} \mathrm{X}$, Zlatava G, Loftus J. Burden of illness, visual impairment and health resource utilisation of patients with neovascular age-related macular degeneration. $\mathrm{Br} \mathrm{J}$ Ophthalmol. 2007;91:1303-7.

11. Sivaprasad S, Hykin P, Chakravarthy U, Lotery A, McKibbin M, Napier J. A retrospective study of the real-life utilization and effectiveness of ranibizumab therapy for neovascular age-related macular degeneration in the UK. Clin Ophthalmol. 2016;10:87-96. 
12. Ashton JR. Sight loss: a public health priority. https://www.rnib. org.uk/sites/default/files/Sight_loss_apublic_health_priority_PT_ 1401.docx. (2009). Accessed 23 Dec 2017.

13. Hiscock R, Bauld L, Amos A, Fidler JA, Munafò M. Socioeconomic status and smoking: a review. Ann N Y Acad Sci. 2012;1248:107-23.

14. Sharma HE, Mathewson PA, Lane M, Shah P, Glover N, Palmer $\mathrm{H}$, et al. The role of social deprivation in severe neovascular age-related macular degeneration. $\mathrm{Br} \mathrm{J}$ Ophthalmol. 2014;98:1625-8.

15. Yip JL, Khawaja AP, Chan MP, Broadway DC, Peto T, Luben R, et al. Area deprivation and age related macular degeneration in the EPIC-Norfolk Eye Study. Public Health. 2015;129:103-9.

16. Acharya N, Lois N, Townend J, Zaher S, Gallagher M, Gavin M. Socio-economic deprivation and visual acuity at presentation in exudative age-related macular degeneration. $\mathrm{Br} \mathrm{J}$ Ophthalmol. 2009;93:627-9.

17. Attebo K, Mitchell P, Smith W. Visual acuity and the causes of visual loss in Australia-The blue mountains eye study. Ophthalmology. 1996;103:357-64.

18. Boulanger-Scemama E, Querques G, About F, Puche N, Srour M, Mane V, et al. Ranibizumab for exudative age-related macular degeneration: A five year study of adherence to followup in a real-life setting. J Fr d Opthalmologie. 2015;38: 620-7.

19. Polat O, İnan S, Özcan S, Doğan M, Küsbeci T, Yavaş GF, et al. Factors affecting compliance to intravitreal anti-vascular endothelial growth factor therapy in patients with age-related macular degeneration. Türk Oftalmol Derg. 2017;47:205-10.

20. Latalska M, Matysik-Woźniak A, Bylina J, Latalski M, Rejdak R, Mackiewicz J, et al. Wet age-related macular degeneration (wet AMD) in rural and urban inhabitants in south-eastern Poland. Ann Agric Environ Med. 2013;20:726-30.

21. Kelly C, Hulme C, Farragher T, Clarke G. Are differences in travel time or distance to healthcare for adults in global north countries associated with an impact on health outcomes? A systematic review. BMJ Open. 2016;6:e13059.

22. NICE. Aflibercept solution for injection for treating wet age-related macular degeneration I Guidance and guidelines I NICE. https://www.nice.org.uk/guidance/ta294. (2018). Accessed 2 May 2018.

23. NICE. Age-related macular degeneration. https://www.nice.org. uk/guidance/gid-cgwave0658/documents/short-version-of-draftguideline-2. Accessed 15 Oct 2017.

24. Almuhtaseb H, Kanavati S, Rufai SR, Lotery AJ. One-year realworld outcomes in patients receiving fixed-dosing aflibercept for neovascular age-related macular degeneration. Eye. 2017;31: 878-83.

25. Dandona L, Dandona R. Revision of visual impairment definitions in the International Statistical Classification of Diseases. BMC Med. 2006;4:7.

26. The English Index of Multiple Deprivation (IMD) 2015-Guidance. https://www.gov.uk/government/statistics/english-indicesof-deprivation-2015. Accessed 15 Oct 2017.

27. Support UDSC. GeoConvert, UK Data Service Census Support. 2015 Jul 29; http://geoconvert.mimas.ac.uk/. Accessed 22 Mar 2018.

28. ArcGIS. The mapping and analytics platform. http://www.esri. com/arcgis/about-arcgis. Accessed 15 Oct 2017.
29. Royal National Institute of Blind People. The criteria for certification. http://www.rnib.org.uk/eye-health/registering-your-sightloss/criteria-certification. (2018). Accessed 22 Mar 2018.

30. Bachmann M, Eachus J, Hopper CD. Socio-economic inequalities in diabetes complications, control, attitudes and health service use: a cross-sectional study. Diabet Med A J Br Diabet Assoc. 2003;20:921-9.

31. Ng W, Agarwal P, Sidiki S, McKay L, Townend J, Azuara-Blanco A. The effect of socio-economic deprivation on severity of glaucoma at presentation. Br J Ophthalmol. 2010;94:85-7.

32. Chua P, Mustafa M, Scott N, Kumarasamy M, Azuara-Blanco A. Relationship between socioeconomic deprivation or urban/rural residence and visual acuity before cataract surgery in northern Scotland. Eur J Ophthalology. 2013;23:831-5.

33. Hamano T, Li X, Tanito M, Nabika T, Shiwaku K, Sundquist J, et al. Neighborhood deprivation and risk of age-related eye diseases: a follow-up study in Sweden. Ophthalmic Epidemiol. 2015;22:308-20.

34. Chew H, Maberley DA, Ma P, Chang A, Maberley A. Socioeconomic status and clinical features of patients undergoing photodynamic therapy or transpupillary thermotherapy for subfoveal choroidal neovascularization due to age-related macular degeneration. Can J Ophthalmol. 2005;40:384-8.

35. Klein R, Klein BE, Jensen SC, Moss SE. The relation of socioeconomic factors to the incidence of early age-related maculopathy: the Beaver Dam eye study. Am J Ophthalmol. 2001;132:128-31.

36. Park SJ, Lee JH, Woo SJ, Ahn J, Shin JP, Song SJ, et al. Agerelated macular degeneration: prevalence and risk factors from Korean National Health and Nutrition Examination Survey, 2008 through 2011. Ophthalmology. 2014;121(9):1756-65.

37. Cackett P, Tay WT, Aung T, Wang JJ, Shankar A, Saw SM, et al. Education, socio-economic status and age-related macular degeneration in Asians: the Singapore Malay Eye Study. Br J Ophthalmol. 2008;92:1312-5.

38. Nicklett EJ, Szanton S, Sun K, Ferucci L, Fried LP, Guralnik JM, et al. Neighborhood socioeconomic status is associated with serum carotenoid concentrations in older, community-dwelling women. J Nutr. 2011;141:284-9.

39. Bernstein P, Delori FC, Richer S, Kuijk FJM, Wenzel A. The value of measurement of macular carotenoid pigment optical densities and distributions in age-related macular degeneration and other retinal disorders. Vision Res. 2010;50:716-28.

40. Cross V, Shah P, Bativala R, ReGAE PS. 2: glaucoma awareness and the primary eye-care service: some perceptions among African Caribbeans in Birmingham UK. Eye. 2007;21:912-20.

41. Protheroe J, Whittle R, Bartlam B, Estacio EV, Clark L, Kurth J. Health literacy, associated lifestyle and demographic factors in adult population of an English city: a cross-sectional survey. Health Expect. 2017;20:112-9.

42. Protheroe J, Nutbeam D, Rowlands G. Health literacy: a necessity for increasing participation in health care. $\mathrm{Br} \mathrm{J}$ Gen Pract. 2009;59:721-3.

43. O'Connor PM, Mu LC, Keeffe JE. Access and utilization of a new low-vision rehabilitation service. Clin Experiment Ophthalmol. 2008;36:547-52.

44. Sloan FA, Yashkin AP, Chen Y. Gaps in receipt of regular eye examinations among medicare beneficiaries diagnosed with diabetes or chronic eye diseases. Ophthalmology. 2014;121:2452-60. 